\section{Multiples Myelom: Proteasom-Inhibitor in der Induktion und Erhaltung}

\begin{abstract}
Hochdosiertes Melphalan mit nachfolgender autologer Stammzelltransplantation gilt als Goldstandard in der Behandlung jüngerer Patienten mit einem multiplen Myelom. Niederländische und belgische Kollegen gingen der Frage nach, ob die Integration des Proteasom-Inhibitors Bortezomib in die Induktions- und Erhaltungstherapie die Behandlung optimiert.
\end{abstract}

\begin{abstract}
$\mathrm{n}$ dieser Open-Label randomisierten Phase-III-Studie wurden 827 Patienten mit neu diagnostiziertem symptomatischem multiplem Myelom behandelt. Die Patienten (median 57 Jahre alt) wurden in zwei Gruppen randomisiert: Induktionstherapie mit Vincristin, Doxorubicin und Dexamethason (VAD) bzw. Bortezomib, Doxorubicin und Dexamethason (PAD) gefolgt von hochdosiertem Melphalan und autologer Stammzelltransplantation. Zur Erhaltungstherapie er-
\end{abstract}

hielt die VAD-Gruppe $50 \mathrm{mg}$ Thalidomid einmal täglich, die PAD-Gruppe 1,3 mg/ $\mathrm{m}^{2}$ Bortezomib einmal 14-tägig über einen Zeitraum von zwei Jahren.

Sowohl in der Induktions- als auch Erhaltungsphase ergaben sich Vorteile für die PAD-Gruppe im Complete Response: insgesamt 36 bzw. $24 \%$. Auch bezüglich des progressionsfreien Überlebens nach einem medianen Follow-up von 41 Monaten war die mit dem Proteasom-Inhibitor behandelte Gruppe dem Vergleichs- arm überlegen (median 35 vs. 28 Monate). Entsprechendes galt auch für das $\mathrm{Ge}$ samtüberleben. Hochrisikopatienten mit erhöhten Kreatininspiegeln profitierten den Ergebnissen zufolge von der Bortezomib-Kombination mit einer von 13 auf 30 Monate mehr als verdoppelten Progressionsfreiheit.

Fazit: Der Proteasom-Inhibitor Bortezomib als Kombinationspartner in der Induktions- und Erhaltungstherapie bei Patienten mit einem neu diagnostizierten multiplen Myelom erhöht die Ansprechrate, das progressionsfreie Krankheitsintervall und verbessert das Gesamtüberleben. Wolfgang Zimmermann

Sonneveld P et al. Bortezomib induction and maintenance treatment in patients with newly diagnosed multiple myeloma: results of the randomized phase III HOVON-65/GMMG-HD4 trial. J Clin Oncol. 2012;30(24):2946-55.

\section{Lenalidomid bereichert Erhaltungstherapie}

Lenalidomid zur Erhaltungstherapie nach autologer Stammzelltransplantation ist bei neu diagnostiziertem Multipem Myelom eine erfolgreiche Therapieoption. In der Studie MM-015 wurde geprüft, ob das auch für Patienten ohne Stammzelltransplantation gilt.

$\mathrm{M}$ elphalan plus Prednison ist seit langem die Standardkombination für Patienten mit Multiplem Myelom, die älter als 65 Jahre sind. Zusätzlich verabreichtes Lenalidomid, das strukturell mit Thalidomid verwandt ist, hat die Therapie bereichert, weil das Präparat tumorzellabtötende und immunmodulatorische Eigenschaften besitzt. In der MM015-Studie wurde die Wirksamkeit der Melphalan-Prednison-Lenalidomid-

Kombination und von Lenalidomid als Erhaltungstherapie geprüft. Vergleichsgruppe waren Patienten, die nur die Dreifachkombination oder Melphalan plus Prednison ohne Erhaltungstherapie erhielten. Die Patienten mit neu diagnostiziertem Myelom waren mindestens 65 Jahre alt und für eine autologe Stammzelltransplantation nicht geeignet.

Insgesamt nahmen fast 460 Patienten in annähernd gleich großen Gruppen an der Studie teil. Sie erhielten neun Therapiezyklen Melphalan/Prednison/Lena- lidomid (q4w) sowie Lenalidomid als Erhaltungstherapie, die Dreifachkombination plus ein Scheinpräparat als Erhaltungstherapie oder nur Melphalan plus Prednison bis zu einem Rezidiv oder zur Krankheitsprogression. Der Follow-up dauerte ein bis 47 Monate (median 30 Monate). Primärer Studienendpunkt war das progressionsfreie Überleben (PFS).

Am meisten profitierten jene Patienten, die die Dreifachkombinationstherapie mit Lenalidomid-Erhaltungstherapie erhielten. Denn bei ihnen lag das PFS bei 31 Monaten und war damit signifikant länger als in der Vergleichsgruppe mit der Dreifachkombi (14 Monate; Hazard Ratio [HR] 0,49; p < 0,001) und als in der Gruppe der Patienten mit Melphalan plus Prednison (13 Monate; HR 0,40; $\mathrm{p}<0,001)$. Der Vorteil beim Parameter "progressionsfreies Überleben“ wurde allerdings nur bei Patienten im Alter zwischen 65 und 75 Jahren beob- achtet, nicht dagegen bei Patienten über 75 Jahre. Die Studienergebnisse bedeuten, dass durch die Erhaltungstherapie mit Lenalidomid eine Reduktion der Progressionsrate um $66 \%$ erreicht wird, und zwar im Vergleich zur Gruppe der Patienten mit der Dreifachkombinationstherapie ohne Erhaltungstherapie (HR 0,34). Eine Aussage zum Gesamtüberleben erlauben die Daten nicht.

Unerwünschter Nebeneffekt der Erhaltungstherapie mit Lenalidomid war die erhöhte Rate an invasiven Zweittumoren (7 vs. $3 \%$ unter Melphalan plus Prednison), doch nach Angabe der Studienautoren überwiegte der Nutzen der Erhaltungstherapie.

Fazit: Myelom-Patienten, für die eine Stammzelltransplantation nicht infrage kommt, profitieren von einer Erhaltungstherapie mit Lenalidomid, weil sich das PFS deutlich verlängert. Zwar gilt das PFS beim Myelom als anerkannter Surrogatparameter für das Gesamtüberleben, doch bleibt für manche die Frage, ob das PFS als primärer Endpunkt in Studien zur Erhaltungstherapie geeignet ist.

Peter Leiner

Palumbo A et al. Continuous lenalidomide treatment for newly diagnosed multiple myeloma. N Engl J Med. 2012;366(19):1759-69. 